\title{
Enunciación
}

http://revistas.udistrital.edu.co/ojs/index.php/enunc

DOI: http://dx.doi.org/10.14483/udistrital.jour.enunc.2016.1.a06

enunciación

Artículo de reflexión

\section{La escritura extensiva. La enseñanza de la expresión escrita en secundaria}

\author{
The extensive writing. Teaching writing in high school \\ Daniel Cassany Comas ${ }^{1}$
}

Para citar este artículo: Cassany, D. (2016). La escritura extensiva. La enseñanza de la expresión escrita en secundaria. Enunciación, 21(1), 91-106.

Recibido: 07-enero-2016 / Aprobado: 16-mayo-2016

\section{Resumen}

Siguiendo la distinción entre lectura intensiva y extensiva, presentamos las tareas extensivas de expresión escrita para fomentar objetivos de aprendizaje como: 1) acercar la escritura a la vida personal del aprendiz; 2) practicar las funciones epistémica y comunicativa del lenguaje; 3 ) ceder al aprendiz toda la responsabilidad del acto creativo; 4) facilitar el desarrollo de los procesos cognitivos, y 5) generar hábitos sostenidos de producción escrita en variedad de situaciones. Por ello, las tareas extensivas de escritura producen escritos extensos, duran un periodo de tiempo largo, son autodirigidas por el aprendiz-autor, tratan temas interdisciplinarios y no forman parte del libro de texto. Dichas tareas difieren notablemente de los ejercicios escritos más frecuentes en el aula, dirigidos por el docente, con textos breves, funciones ejecutivas o instrumentales y una corrección centrada en la ortografía y la gramática. Proponemos varios instrumentos didácticos para fomentar este tipo de tareas: carpeta o portafolios (para guardar borradores, correcciones y versiones finales de cada texto), formatos (como diarios de lectura, apuntes de clase o protocolos de laboratorio) y contextos (tareas comunicativas del día a día). También argumentamos a favor de algunos parámetros básicos de las tareas extensivas, como la extensión del texto, el uso de varias sesiones de trabajo para la producción, la práctica de los procesos y subprocesos de composición o el uso de la revisión entre iguales, en parejas o equipos.

Palabras clave: escritura, enseñanza de la escritura, didáctica, aprendizaje activo.

\begin{abstract}
Following the distinction between intensive and extensive reading, we introduce the extensive written tasks to promote the following learning objectives: 1) bringing writing closer to the learner's personal life; 2) practicing the epistemic and communicative language functions; 3) giving the learner full responsibility for the creative act; 4) facilitating the development of cognitive processes, and 5) developing habits of written production in a variety of situations. As a consequence, extensive writing assignments produce longer texts, last longer, are self-directed by the learner-author, treat interdisciplinary topics and are not in the textbook. These tasks markedly differ from the more frequent written exercises in the classroom, which are teacher-led, contain shorter texts, work on executive
\end{abstract}

1 Universitat Pompeu Fabra. Correo electrónico: daniel.cassany@upf.edu 
or instrumental functions, and their correction is focused on spelling and grammar. We propose several educational tools in order to develop this type of tasks: portfolios (to save drafts, corrections and final versions of each text), formats (such as reading logs, lecture notes and laboratory protocols) and contexts (common communicative tasks). We also discuss some basic parameters of extensive tasks, such as the length of the text, the use of several working sessions for text production, the practice of composition processes and the use of peer review, in pairs or teams.

Keywords: writing, teaching writing, didactics, active learning.

a quién, de qué manera; con qué estilo; etc. La ausencia de actividades extensivas de redacción, con las cuales el aprendiz adquiera más autoridad sobre la escritura, puede ser -a mi modo de ver- una de las causas de la sensible desvirtuación que padece la práctica de la escritura en secundaria. Los aprendices se sienten desmotivados, perezosos o aburridos, cuando tienen que escribir, precisamente porque no pueden experimentar la libertad que requiere la práctica de la escritura.

Mediante un paralelismo entre lectura y escritura, esta ponencia presenta un enfoque extensivo de la expresión escrita, el cual pretende conseguir objetivos didácticos actitudinales (generar interés y motivación para escribir, fomentar hábitos de escritura, aproximar la escritura a la vida personal, etc.) $y$, en definitiva, restituir a los aprendices la libertad que les pertenece como autores. Los apartados de la ponencia definen los conceptos de actividad intensiva y extensiva, describen los objetivos de un enfoque extensivo, comentan sus características didácticas (organización, tipo de texto, evaluación) y presenta varios ejemplos didácticos.

\section{ACTIVIDADES INTENSIVAS Y EXTENSIVAS}

La distinción entre lectura intensiva y extensiva es bastante conocida en la enseñanza de segundas lenguas, especialmente en inglés. Se refieren a ella, entre otros, Munby (1968), White (1983), y Ribé y Dejuan (1984). A partir de estos autores y también de Cassany, Luna y Sanz (1993), se pueden establecer las siguientes distinciones, necesariamente orientativas y genéricas (tabla 1). 
Tabla 1. Características de las actividades intensivas y extensivas

\begin{tabular}{ll}
\hline \multicolumn{1}{c}{ Actividades intensivas } & \multicolumn{1}{c}{ Actividades extensivas } \\
\hline Tienen objetivos de orden conceptual o procedimental. & Tienen objetivos de orden actitudinal. \\
$\begin{array}{l}\text { Trabajan con textos breves (fragmentos, párrafos, una } \\
\text { página, un cuento, etc.). }\end{array}$ & $\begin{array}{l}\text { Trabajan con textos más extensos, sin límites (libros, diarios } \\
\text { personales, etc.). } \\
\text { Tienen poca duración (minutos, una hora, una sesión). } \\
\text { Tienen larga duración (semanas, meses, un trimestre o incluso } \\
\text { un curso). }\end{array}$ \\
$\begin{array}{ll}\text { Suelen ser heterodirigidas: el profesor las diseña, gestio- } \\
\text { na y evalúa. }\end{array}$ & $\begin{array}{l}\text { Son más autodirigidas: el aprendiz asume parte de autonomía } \\
\text { y responsabilidad. }\end{array}$ \\
$\begin{array}{l}\text { Suelen ser disciplinarias del área de lengua. } \\
\text { Suelen estar relacionadas con el libro de texto. }\end{array}$ & Se apartan del él. \\
\hline
\end{tabular}

Fuente: elaboración propia.

Las actividades intensivas se plantean objetivos de orden conceptual (aprender un determinado ítem lingüístico: un campo léxico, una estructura sintáctica o discursiva, etc.) o procedimental (practicar alguna destreza específica: anticipación de información, inferencia de datos, etc.) a partir del trabajo con textos breves durante un tiempo preestablecido, con la mediación directa del profesorado. Suelen ser actividades exclusivamente lingüísticas que no incorporan contenidos de otras áreas y que, en muchos casos, proceden del libro de texto.

En cambio, las actividades intensivas se plantean principalmente objetivos de tipo actitudinal (fomentar hábitos, cambiar actitudes, desarrollar motivación, etc.) a partir de la utilización de textos extensos durante un periodo dilatado de tiempo. Suelen dar más protagonismo al aprendiz, aunque sea el profesor el que proponga y organice la actividad. También pueden integrar contenidos de materias no lingüísticas, pasando a ser ejercicios transversales o transcurriculares, de modo que se independizan de la estrecha programación del libro de texto.

Los términos heterodirigido y autodirigido proceden de estudios sobre autonomía y autoaprendizaje en enseñanza de segundas lenguas (Bosch, 1996; Holec, 1988). El primero denomina los ejercicios que gestiona totalmente una persona distinta del sujeto que aprende (sea esta el profesor del aula o el pedagogo y lingüista, autores del método de enseñanza programada que sigue un autodidacta en una biblioteca o en su casa) ${ }^{2}$. El segundo término se refiere a los materiales didácticos, preparados por un profesor o un pedagogo, en los que el aprendiz puede asumir responsabilidades sobre su propio proceso de aprendizaje: puede decidir qué quiere aprender, con qué actividades, cómo quiere resolverlas, etc. En general, en las actividades extensivas el aprendiz ejerce un grado de autonomía muy superior al que puede asumir en las intensivas, lo cual permite que pueda desarrollar actitudes e intereses positivos respecto al objetivo de aprendizaje.

Un análisis rápido de las actividades de comprensión lectora que se realizan con regularidad en una clase de lengua de secundaria permite distinguir con facilidad entre los dos grupos. Por un lado, son claramente intensivos las lecturas guiadas con preguntas previas o finales, el comentario de textos (literarios o no), un cloze (texto con vacíos léxicos que deben rellenarse), o una sopa de párrafos procedentes de diferentes escritos, que deben clasificarse y ordenarse. Por otro lado, son extensivas todas las prácticas encaminadas a fomentar la lectura extraescolar de libros (lecturas de curso [novelas,

2 De manera que enseñanza heterodirigida no coincide necesariamente con enseñanza presencial, ni tampoco aprendizaje autodirigido coincide con enseñanza a distancia. La autodirección, que incluye la autonomía en el aprendizaje y el autoaprendizaje, es más bien una conducta que el aprendiz desarrolla respecto a su propio proceso de aprendizaje, al margen que el aprendiz asista o no a clase. 
ensayos, artículos, etc.], consultas en la biblioteca, visitas a ferias de libros, charlas con autores, debates sobre libros leídos, etc.).

Si intentamos realizar un análisis similar con las prácticas de escritura, descubriremos una realidad muy distinta. La inmensa mayoría de los ejercicios de redacción son de carácter intensivo, ya sean instrucciones de los libros de texto que el profesorado actualiza y adapta a cada curso (comentarios de texto, ensayos, redacción de temas más o menos previsibles), o actividades preparadas al margen de aquél (cartas, postales, instancias, etc.). También deben considerarse intensivas las prácticas más académicas de redacción, como la toma de apuntes, el resumen de artículos o libros, los esquemas e, incluso, los exámenes de todas las materias, lingüísticas y no lingüísticas, que realiza el aprendiz $z^{3}$.

Estas prácticas de escritura comparten una serie de características didácticas que, al no poder contrastarse con otra clase de actividades más extensivas (tal como ocurría con la enseñanza de la lectura), ejercen una influencia negativa sobre las actitudes que los aprendices desarrollan hacia la escritura. Entre otros factores perniciosos, cabe destacar los cuatro siguientes:

- El profesorado gestiona, de forma directa, las prácticas, de modo que el aprendiz puede tomar poca iniciativa personal. El aprendiz raramente puede decidir lo que quiere escribir (temas, tipo de texto) o cómo quiere hacerlo (tono, estilo, etc.).

- La mayoría de escritos que produce el aprendiz son textos breves dirigidos al profesorado. Aunque se propongan contextos verosímiles de escritura (una carta dirigida al

3 Seguramente cada día son más frecuentes en secundaria, sobre todo en las materias de idioma, actividades más creativas de escritura -como el texto libre freinetiano (Freinet, 1968, 1969): con mucha tradición en primaria en Catalunya-, el taller de textos literarios (Sánchez y Rincón, 1985) o sociales, los proyectos de escritura (con un grado discutible de autodirección), o la elaboración de programas en los medios de comunicación (prensa y radio), por poner solo algunos ejemplos. Pero parece difícil creer que este tipo de prácticas puedan igualar o superar en cantidad y calidad a las intensivas mencionadas más arriba (ver también nota 4). ayuntamiento para pedir una subvención), el aprendiz no acaba de creerse la situación y el contexto académico real (un escrito para el profesor para sacar buena nota) se impone sobre ficticio. (En la sección "Contextos" se analizan las limitaciones cognitivas y lingüísticas que imponen este tipo de textos.)

- La mayoría de escritos que produce el aprendiz tienen función ejecutiva o instrumental (y en algunos pocos casos funcional) de acuerdo con los cuatro niveles estratificados de dominio escolar de la escritura, propuestos por Wells $(1987)^{4}$. Se prescinde de los usos epistémicos, en los que el usuario puede escribir para aprender, estudiar, crear o imaginar.

- La evaluación (corrección) de la práctica suele basarse exclusivamente en la calidad lingüística del producto final. No se tienen en cuenta el proceso de composición del texto (estrategias cognitivas, técnicas de redacción) ni las actitudes generales que muestra el aprendiz ante la escritura. Por otra parte, el profesorado impone unos determinados criterios de éxito (corrección, gramaticalidad) sin que haya habido una negociación previa de los mismos entre las partes interesadas. En consecuencia, el aprendiz acaba escribiendo lo que su docente quiere que escriba: se produce un claro fenómeno de apropiación del texto (Reid, 1994, p. 275) por parte del profesorado, en el que el aprendiz renuncia a sus derechos legítimos de autor del texto.

En resumen, la preponderancia absoluta de actividades intensivas de escritura fomenta una enseñanza gramatical y heterodirigida, y desatiende el

4 Wells (1987) distingue los cuatro niveles siguientes: nivel ejecutivo, en el que escribir es solo una operación mecánica de transcripción de la oralidad; nivel funcional, en el que se usa la escritura para comunicarse entre personas que están en sitios diferentes; nivel instrumental, en el que escribimos lo que queremos guardar o transformar (resumir, comparar, anotar, etc.), y nivel epistémico, en el que escribimos para crear conocimiento nuevo, para aprender. 
componente actitudinal (valores, opiniones, sentimientos, motivación) de la educación lingüística. Con estos planteamientos, resulta muy difícil que un aprendiz pueda desarrollar actitudes positivas hacia la expresión escrita: que aprenda a usar la escritura espontánea y libremente para conseguir todo tipo de objetivos personales, académicos, sociales o profesionales.

\section{OBJETIVOS DEL ENFOQUE EXTENSIVO}

La introducción de un enfoque extensivo de la escritura, el cual-como vimos con la lectura- resulta compatible con los ejercicios intensivos, permite enderezar los aspectos criticados en el apartado anterior. He aquí los principales objetivos que se plantean las actividades extensivas de redacción:

1. Permitir que el aprendiz asuma la responsabilidad íntegra de la producción de textos, la cual le pertenece legítimamente como autor.

2. Acercar la práctica de la escritura a la vida personal del aprendiz, de modo que escriba para todo tipo de finalidades y en todo tipo de contextos.

3. Formar los aprendices en los procesos de composición de textos: planificación, textualización y revisión, y en el control metacognitivo de todo el proceso.

4. Aprender a usar la escritura en todas sus funciones (ver nota 4) y sobre todo como instrumento académico de trabajo (guardar datos, manipular información, estudiar, aprender, etc.) y de creación de conocimiento (reflexión, crítica, etc.).

5. Desarrollar la iniciativa personal en la escritura.

6. Formar el hábito de escribir de manera continuada, sin necesidad de motivación externa o instrucción directa del profesor.

En conjunto, estos objetivos exigen un cambio muy importante de los roles (o funciones) que hasta hoy han mantenido profesorado y aprendices. El primero tiene que dejar de fiscalizar las prácticas de escritura para convertirse en un simple asesor técnico del segundo. Este debe asumir las responsabilidades que le pertenecen como auténtico protagonista del proceso de aprendizaje de la escritura. En resumen, la pareja profesor/alumno tiene que entenderse como la manifestación escolar de la relación básica que se establece entre autor y lector, con los derechos y los deberes típicos de esta situación. La tabla 2 resume algunos de los derechos y deberes fundamentales de ambos.

Tabla 2. Derechos y deberes del aprendiz-autor y del profesor-lector

\begin{tabular}{ll}
\hline \multicolumn{1}{c}{ Aprendiz-autor } & \multicolumn{1}{c}{ Profesor-lector } \\
\hline Poder escribir lo que quiera, cuando quiera y como quiera. & Negociar con el autor las características de lo que escribirá. \\
Responsabilizarse de la calidad de sus textos. & Dar un margen razonable de confianza al autor. \\
$\begin{array}{ll}\text { Utilizar todo tipo de recursos (ordenadores, diccionarios, } \\
\text { gramáticas, etc.) para escribir. }\end{array}$ & Aconsejar al autor para que pueda mejorar su texto \\
Asumir la corrección y la mejora de sus textos. & $\begin{array}{l}\text { Respetar el texto del autor (opiniones, punto de vista, estilo, } \\
\text { etc.). No apropiarse de este. }\end{array}$ \\
\hline
\end{tabular}

Fuente: elaboración propia. 
En resumen, esta nueva concepción de los roles de profesor y de aprendiz restituye las funciones legítimas que cada uno debe ejercer en un proceso comunicativo escrito como es el de la clase de lengua. El aprendiz-autor debe encargarse de buscar temas de escritura (previamente negociados con el profesor-lector), debe responsabilizarse de la calidad de su escrito y debe asumir la tarea continuada de corregirlo. En cambio, el profesor debe tratar el texto de su aprendiz del mismo modo que trataría el de un profesional: con el mismo respeto y margen de confianza; también debe aconsejar a su autor sobre cómo puede mejorar el texto.

\section{LA CARPETA COMO ENTORNO}

Sin duda las actividades extensivas de escritura, así como los roles de aprendiz-autor y profesor-lector mencionados, no pueden desarrollarse en el contexto tradicional de la clase de Lengua, con las pautas habituales que se siguen en la clase (lección magistral, resolución de ejercicios, etc.). Este nuevo enfoque didáctico de la escritura requiere un entorno escolar nuevo, una nueva organización, que permita las novedades que hemos apuntado hasta ahora.

La carpeta (conocida como portfolio en inglés) constituye el marco idóneo en el cual desarrollar este nuevo enfoque. Se trata de un archivo individual de todos los trabajos de redacción realizados durante el curso. El aprendiz tiene que guardar el protocolo completo (anotaciones, listas, esquemas, borradores, correcciones, versión final, etc.) de todas las prácticas de escritura (apuntes, redacciones, resúmenes, exámenes, trabajos, etc.) en un pequeño archivo de materiales (carpeta, libreta, portafolios) durante todo el curso académico -o incluso durante un ciclo completo (ESO)-. De esta manera, la carpeta se convierte en un registro completo de todos los textos que produce el aprendiz y en un espejo detallado de toda la actividad realizada en clase (temas tratados, discusiones, redacciones, correcciones, etc.).

Las funciones que puede ejercer la carpeta durante el curso son muy variadas. Para el aprendiz, al inicio resulta una manera fácil de aprender a guardar y valorar lo que se escribe; más adelante, cuando empieza a tener bastante material, se convierte en una fuente inagotable de ideas, sugerencias o textos corregidos que se pueden consultar y reutilizar en otras circunstancias. Para el profesor, es un instrumento fundamental para evaluar los procesos de composición, los hábitos y las actitudes y los valores que tiene el aprendiz respecto a la escritura; le permite diagnosticar las dificultades que tiene el sujeto, verificar su progreso o sugerir qué tipo de actividades (técnicas, tipos de texto, prácticas, etc.) pueden serle más útiles. Para ambos, aprendiz y profesor, la carpeta constituye un sistema de organización de la clase muy asequible y con grandes posibilidades.

Para llevar a cabo con éxito la organización de las carpetas, el aprendiz debe seguir unas reglas organizativas básicas (ver también Cassany, 1993a) (tabla 3).

En resumen, la carpeta ofrece un entorno constructivo para fomentar la escritura extensiva: permite que aprendiz y profesor desarrollen sus roles de lector y autor; trata con el mismo valor las versiones finales y los borradores intermedios -de manera que el aprendiz aprende a valorar estos últimos-; construye un nutrido archivo de materiales que puede ser objeto de análisis y aprovechamiento posterior, y transmite la concepción de la escritura como un instrumento multifuncional que acompaña permanente a los aprendices y al profesorado ${ }^{5}$.

5 La bibliografía norteamericana sobre portafolios es inagotable (Murphy y Smith, 1992; Gill, 1993). En España se publicaron algunos artículos norteamericanos en CLE, 19-20 (Calfee y Perfumo, 1993; Valencia, 1993). 
Tabla 3. Reglas de la carpeta

- Queda prohibido tirar cualquier escrito, sea completo o fragmentario, intermedio o final. El aprendiz tiene que guardar todo lo que produce para la asignatura de Lengua.

- Cada documento constituye una entrada de la carpeta y cada entrada debe incluir un título que identifique el texto y la fecha de su elaboración*.

- Cada trabajo de redacción debe contener el protocolo completo del proceso de composición (borradores, notas, revisiones, versión final pasada en limpio, etc.), ordenado cronológicamente.

- Aunque el profesor proponga ejercicios variados de redacción (ver apartado "Tareas de escritura"), el aprendiz tiene libertad absoluta para tomar la iniciativa y hacer todo tipo de entradas en su carpeta. La evaluación periódica de la carpeta valorará el número de entradas, así como sus características (ver apartado "Evaluación").

- Durante el curso se pueden -y deben- realizar distintas actividades a partir de la carpeta, como: analizar las producciones realizadas hasta el momento, elaborar instrumentos variados de redacción que ayuden al aprendiz a escribir (listas de vocabulario difícil, listas con los errores más frecuentes que comente, chuletas** con los marcadores discursivos más útiles, etc.). Este tipo de ejercicios anima al aprendiz a usar su carpeta como un verdadero escritorio que facilita la tarea de producir textos.

- Profesor y aprendiz realizan periódicamente (cada evaluación formal, cada mes o cada trimestre) una evaluación formativa de la carpeta, que consiste en analizar el material producido según varios criterios (ver apartado "Evaluación").

Los pedagogos que han analizado críticamente el método de las carpetas, han destacado las siguientes características pedagógicas, muy beneficiosas para el aprendiz (Cassany, 1993a):

- Tiene el aprendiz como protagonista o centro (el profesor no lo dirige).

- Está orientado a desarrollar el proceso de composición (y no el producto).

- Relaciona la escritura con los temas del currículum y con la experiencia de cada uno.

- Busca la colaboración entre aprendices y entre aprendices y profesor.

- Favorece la autonomía y la responsabilidad del aprendiz.

- Es muy flexible y se puede utilizar con varias finalidades.

- Permite realizar una coevaluación formativa.

* El concepto de entrada es relativo: son entradas distintas en los apuntes de una sesión, el resumen de un escrito, los comentarios de un texto, etc., pero también pueden constituir una única entrada el conjunto numeroso y variado de notas y borradores que se generan durante el proceso de composición de un único texto.

** El DRAE define chuleta en las acepciones segunda y tercera como: "apunte que se lleva oculto para copiar en los exámenes" y "nota o conjunto de notas que utiliza quien habla en público para recordar algunos puntos de su exposición", respectivamente. Dicho concepto recibe otras denominaciones según los países hispanos: acordeón en México; comprimido en Perú; torpedo en Chile;machete en Argentina; ferrocarril o trencito en Uruguay; chivo en Cuba; chancuco, copialina o pastel en Colombia (Wikilengua y Wordreference).

Fuente: elaboración propia.

\section{TAREAS DE ESCRITURA}

La selección de las tareas de escritura (o de las consignas, instrucciones o contextos de expresión escrita) es otra cuestión fundamental de la organización de clase. Las actividades extensivas tienen especificidades importantes que afectan a la concepción de las tareas, al grado de implicación del aprendiz y a los tipos de texto que se escriben. En principio, debemos distinguir entre dos tipos básicos de tareas, con objetivos y características muy diferentes: los formatos y los contextos de escritura. La tabla 4 presenta las principales diferencias entre estos dos tipos de actividades. 
Tabla 4. Formatos y contextos de escritura

\begin{tabular}{ll}
\hline \multicolumn{1}{c}{ Formatos } & \multicolumn{1}{c}{ Contextos } \\
\hline $\begin{array}{l}\text { Son instrucciones muy generales de redacción. Carecen } \\
\text { de destinatario y tienen propósitos y tipos de texto muy } \\
\text { débiles. }\end{array}$ & $\begin{array}{l}\text { Son situaciones reales o verosímiles de comunicación } \\
\text { que incluyen destinatario, propósito, tipo de texto, marco } \\
\text { compartido autor-lector, etc. } \\
\text { Tienen el objetivo de fomentar la escritura continuada. } \\
\text { Tienen el objetivo de fomentar la capacidad de adapta- } \\
\text { ción del aprendiz a diversas situaciones comunicativas, } \\
\text { con restricciones variadas. } \\
\text { Enfatizar en la función comunicativa (Wells, 1987). }\end{array}$ \\
$\begin{array}{l}\text { Ponen énfasis en las funciones epistémica (Wells, 1987) y } \\
\text { expresiva Jakobson, 1963). }\end{array}$ & $\begin{array}{l}\text { Los criterios con que se evalúan se refieren al conte- } \\
\text { nos criterios con que se evalúan se refieren sobre todo forma: coherencia, cohesión, adecuación, } \\
\text { corrección, etc. }\end{array}$ \\
$\begin{array}{l}\text { la contenido del escrito: la cantidad de texto producido, } \\
\text { reflexión y personalización, etc. }\end{array}$ & \\
\hline
\end{tabular}

Fuente: elaboración propia.

A continuación se presenta, comenta y ejemplifica cada tipo.

\section{Formatos}

El formato tiene el objetivo fundamental de facilitar que el aprendiz descubra cómo la escritura puede ser un instrumento útil para investigar sus ideas, para estudiar o para reflexionar, en los ámbitos personal, académico y profesional. Intenta conseguir que, de acuerdo con sus intereses y necesidades, el aprendiz integre individualmente la escritura en su actividad cotidiana, del mismo modo que puede utilizar la lectura, la conversación o el estudio (válida para aplicar en numerosas cuestiones académicas, personales o profesionales). Para conseguirlo, el formato debe ser una instrucción extremadamente flexible y adaptable a todo tipo de intereses particulares, y debe crear el hábito periódico y continuado de escribir.

Los formatos suelen ser instrucciones muy genéricas que exigen que el aprendiz escriba periódicamente durante un largo lapso de tiempo (meses, un trimestre, un curso completo). Acostumbran a tener un tema más o menos específico de punto de partida (una lectura, un viaje, una experiencia), pero permiten $-y$ animan a- que el aprendiz desarrolle sus ideas y las dirija hacia campos más personales de su interés, alejados del foco inicial de escritura. La evaluación de las producciones escritas del formato se basa exclusivamente en la cantidad de texto producido, en el número de sesiones de que consta o en la diversidad de temas que trata; el profesor no corrige con los criterios lingüísticos habituales, puesto que las producciones no son auténticos textos comunicativos, dirigidos a un lector-de hecho, muchas veces el profesor no dispone del tiempo suficiente para leer la enorme cantidad de producciones que genera el aprendiz- ${ }^{6}$.

Son ejemplos de formatos extensivos de escritura las siguientes actividades:

- Diarios. A partir del formato tradicional de diario (distintas entradas con fechas ordenadas cronológicamente), el aprendiz escribe periódicamente sobre todo tipo de temas. Puede tratarse de un diario personal o íntimo, a la usanza tradicional, o de diarios específicos de una lectura, un viaje, una actividad de clase o una experiencia.

6 Algunos profesores y teóricos sostienen que el profesor no debe leer las producciones del aprendiz (realizadas a partir de actividades de formato), porque inhiben a este último. El alumno escribe de manera muy distinta si sabe que el profesor va a leer lo que escribe. Solo cuando sabe que nadie, excepto él o ella, tendrá acceso a sus escritos, el aprendiz se siente cómodo y relajado, de manera que puede llegar a integrar la práctica de la escritura en su actividad cotidiana. 
- Protocolos. A partir del formato de registro o informe científico de un experimento (distintas entradas con fecha, descripciones objetivas de fenómenos empíricos, formulación de hipótesis de trabajo, enumeración de pasos metodológicos a seguir, etc.), el aprendiz anota todas las incidencias de algún tipo de experimento o actividad que esté realizando para las materias de experimentales o sociales?

- Apuntes. A partir de la actividad tradicional de tomar apuntes, se puede diseñar un formato de escritura menos mecánico (esquemas; ideas importantes con opiniones o dudas personales) que permita al aprendiz escribir y recoger ideas de manera más personal y creativa.
- Bitácora. A partir de la metáfora de la bitácora de barco (el diario de abordo en el que el capitán anota todas las incidencias de la travesía náutica hasta llegar a destino) y usando también el formato de diario, el aprendiz escribe periódicamente sobre sus estudios, sobre las clases, sobre su proceso de aprendizaje (se entiende que el viaje en barco es su curso académico). Se trata de un ejercicio de reflexión sobre la propia actividad de enseñanza/aprendizaje, que intenta fomentar la capacidad de autoanálisis, la responsabilidad y, en definitiva, la autonomía del aprendiz. Los temas de la bitácora pueden estar relacionados con las clases de una asignatura o con unos contenidos determinados.

Tabla 5. Formato de diario de lectura

\section{Diario de lectura}

Diario de lectura es un ejercicio de escritura sobre la lectura de un texto (un libro, unos poemas, un fragmento narrativo, etc.). Se trata de escribir periódicamente sobre las reacciones que te provoque la lectura de un texto. Es una manera de reflexionar con más atención sobre lo que lees: de retener las ideas principales o los pensamientos y las sensaciones que te suscite.

¿Cuántas veces, al leer un texto, se te han ocurrido ideas divertidas, nuevas, distintas... que luego se han perdido porque no las apuntaste a tiempo? ¿Cuántas veces te has aburrido, molestado, cansado, sorprendido, divertido o entusiasmado leyendo un fragmento, y no lo has podido explicar a nadie? Diario de lectura es una forma de recoger todas estas reacciones. Sigue las instrucciones siguientes:

1. Escribe en una libreta pequeña o en hojas sueltas que puedas guardar con el texto que lees.

2. Pon siempre la fecha a cada fragmento que escribas.

3. Empieza el diario con este título: Diario de lectura de... (y el título del texto).

4. Escribe todo lo que te sugiera la lectura: los personajes, el ambiente, el estilo, el argumento, las ideas... Explica si te gustan o no, por qué, qué fragmentos te han interesado más, cuáles menos, etc. Déjate llevar y escribe sobre lo que quieras, sobre lo que te suscite la lectura. De momento, no te preocupes por la ortografía ni por la gramática. Lo importante es conseguir ideas y escritura.

5. Intenta escribir a menudo, cada vez que leas un fragmento del texto. Combina la lectura con la escritura. Puedes apuntar en el diario las citas o las frases que te hayan gustado.

Cuando termines la lectura y el diario, puedes utilizar el material para un pequeño trabajo. Relee tu diario, selecciona los mejores fragmentos, escribe una valoración final, estructúralo todo, corrige la ortografía y ponle el título de Diario de un(a) lector(a). Tendrás un bonito trabajo de lectura/redacción.

Fuente: elaboración propia.

7 Este tipo de formatos permite usar la escritura como instrumento epistémico y regulador en áreas no lingüísticas, siguiendo los principios del movimiento norteamericano de escritura transcurricular Writing across the Curriculum (Escritura a través del currículum) (cf. Fulwiler, 1987; Fulwiler y Young, 1990). 
En la tabla 5 se desarrolla una de las ideas anteriores.

Este tipo de formato utiliza la lectura de un texto (seleccionada por el profesorado o por el aprendiz) como trampolín para fomentar la expresión. El aprendiz debe intercalar constantemente la lectura de un fragmento de texto con la redacción de las reacciones que le suscita. De este modo, la lectura fomenta la escritura y ésta de nuevo anima a la lectura.

\section{Contextos}

Los contextos son tareas de escritura más cercanos a los ejercicios tradicionales y a las actividades intensivas. Como su denominación indica, se trata de contextos comunicativos en los que el aprendiz debe adaptarse a unas circunstancias específicas (propósito, destinatario, tipo de texto, etc.). Las características idóneas de este tipo de actividades son:

- Contextualización. La actividad debe constituir una situación real (o verosímil) de escritura, en la que el texto debe cumplir un conjunto de características lingüísticas (coherencia, cohesión, adecuación, etc.) para tener eficacia real. Debe haber un destinatario concreto, un propósito comunicativo, un tipo de texto y un marco general de referencia entre autor y lector. Además, es importante que las situaciones propuestas coincidan con las necesidades y los intereses del aprendiz (temas, propósitos comunicativos, etc.). Siempre que sea posible, es mucho mejor que la situación sea real (que se trate de un contexto existente y cercano al alumno) y no solo verosímil (como se mencionó más arriba); en este caso el contexto académico se impone al virtual.

- Diversificación. La diversidad de contextos de escritura enriquece las prácticas y permite que el aprendiz desarrolle procesos cognitivos. Los escritos dirigidos a diferentes destinatarios (un periódico, la familia, los compañeros de un curso inferior, etc.) exigen que los aprendices analicen el destinatario que tendrá su texto y el valor que llegará a tener para éste la información que están redactando. Este hecho fomenta la comprensión del proceso comunicativo de la escritura y el desarrollo del proceso de composición.

- Personalización. El aprendiz tiene que escribir sobre sus ideas y opiniones personales (o sobre cualquier tema, pero siempre desde su punto de vista), de manera que tenga que elaborar el contenido del texto conceptual y lingüísticamente. Debemos evitar las consignas de redacción de ensayos y monografías de contenido académico, que acaban convirtiéndose en ejercicios mecánicos de copia y paráfrasis de las enciclopedias, los manuales y los libros de texto.

\section{Extensión}

Un aspecto importante que debe tenerse en cuenta en las actividades de escritura, tanto en los formatos como en los contextos, es la extensión del escrito que produce el aprendiz. La mayoría de ejercicios de los libros de texto, así como las instrucciones que propone el profesor, requieren del aprendiz que produzca textos breves de no más de una o dos páginas (a veces incluso menos: 200-250 palabras, media página, uno o dos párrafos) ${ }^{8}$. Este tipo de escritos favorece un determinado comportamiento escritor del aprendiz que resulta negativo para el desarrollo de sus destrezas cognitivas y de sus actitudes. Veámoslo en los puntos siguientes:

8 Las prácticas de elaboración de escritos más extensos (de 5, 10 o más páginas), como trabajos monográficos, comentarios de libros de lectura, ensayos, etc., cada día son más frecuentes, sobre todo en secundaria y en las materias de letras, pero creo que todavía son inferiores a los ejercicios que utilizan textos breves. Por otra parte, algunas de estas actividades se convierten a menudo en una simple tarea de copia, recorte y pega de los originales (enciclopedias, manuales, libros de texto), por lo que no constituyen auténticas prácticas de expresión. 
- Una sesión de redacción. Cuando la tarea de escritura pide un texto breve, el aprendiz puede producirlo fácilmente en una única sesión de trabajo (una clase de una hora o hora y media, una sesión de media tarde). Este simple hecho limita las posibilidades de reflexión sobre el texto (el autor da por terminado su escrito) e impide que pueda haber un periodo de reposo del texto al que pueda seguir una revisión hecha desde la perspectiva del paso del tiempo.

- Escaso esfuerzo cognitivo. Los textos breves no exigen grandes esfuerzos cognitivos al alumno, que tiende a apuntar en su escrito las primeras ideas que se le ocurren en el orden en que se le ocurren, sin más preocupación sobre si estas son realmente las más relevantes o sobre si están estructuradas de la forma más idónea. Por ejemplo, a un texto de 200-300 palabras le bastan solo dos o tres ideas, si consideramos que este empezará con una introducción del tema y que terminará con una conclusión que repetirá alguna de las ideas expuestas (a parte que los escritos académicos no tienen especial densidad informativa). Naturalmente, ante estas circunstancias el aprendiz raramente tiene dificultades para encontrar ideas y pocas veces experimenta la sensación de tener que buscar ideas para escribir. Además, en el caso que las ideas seleccionadas sean poco relevantes, claras o estén mal ordenadas, el lector no tendrá problemas para entenderlas ya que, al tratarse de textos tan breves, puede reconstruir fácilmente las intenciones originales del autor.

- Esfuerzo lingüístico limitado. Del mismo modo, los escritos breves ofrecen poca dificultad lingüística. Cuestiones como la distribución de la información en capítulos y apartados (¡o incluso párrafos!), la progresión temática (relación entre datos conocidos y datos nuevos), el uso de marcadores discursivos para organizar el texto, o los procedimientos de cita, no suelen tener presencia en los textos breves, de manera que el aprendiz-autor no tiene que enfrentarse a ellos. Del mismo modo, otros aspectos como las relaciones anafóricas entre correferentes alejados (pronombres, elipsis, evitar repeticiones, etc.), la capacidad de diversificar la sintaxis o la elección de un léxico preciso, homogéneo y diversificado a lo largo del texto (terminología, evitar repeticiones, mostrar riqueza expresiva, etc.), tienen mucha menos incidencia en un texto de una cara que en otro de cinco páginas. En resumen, al escribir solo textos breves el aprendiz evita tener que resolver algunas de las cuestiones lingüísticas más importantes que se presentan en la redacción de textos reales. De esta manera, al no haber otros puntos que resolver, el aprendiz concentra su esfuerzo en los aspectos más superficiales de la ortografía y la gramática.

Al contrario, los textos más extensos enfrentan al aprendiz ante una situación y unas exigencias lingüísticas muy superiores que redundan de forma positiva en su comportamiento escritor. Así:

- Varias sesiones de redacción. Las exigencias del escrito extenso (páginas, número de informaciones, estructura, etc.) provocan que los aprendices requieran varias sesiones de trabajo para completar el proceso de composición. El simple hecho de tener que interrumpir y retomar repetidamente este proceso favorece que el aprendiz reflexione más veces sobre su texto, que tenga que releerlo varias veces (como mínimo al inicio de cada nueva sesión de trabajo para recordar lo que había redactado en sesiones anteriores), y que tenga que actualizar el texto de manera constante. El paso del tiempo también favorece la revisión crítica y la madurez. 
- Requerimientos cognitivos complejos. Los escritos extensos exigen un trabajo más importante de elaboración del significado del texto. El aprendiz no dispone espontáneamente de toda la información necesaria y tiene que utilizar distintos procedimientos formales de búsqueda y estructuración de la información. Por otra parte, la extensión del texto requiere una selección y una organización de informaciones muy acorde con las circunstancias comunicativas y con el lector. Este no va a ser tan tolerante con un texto de 5 o 6 páginas, como lo era con uno breve, de modo que el aprendiz-autor tiene que adoptar su punto de vista para construir semánticamente el texto.

- Notable dificultad lingüística. Del mismo modo, los textos extensos presentan algunas características lingüísticas (estructura y progresión de la información, diversificación sintáctica, etc.) de mayor dificultad que las que se incluyen en los textos breves.

En resumen, los textos extensos permiten que el aprendiz sienta la necesidad de dominar los procesos de planificación (generación de ideas, organización) y fomentan también la revisión, con lo cual dilatan el proceso completo de redacción en distintas sesiones. Por todas estas razones, resulta más rentable didáctica y lingüísticamente que el aprendiz escriba un solo texto de 10 páginas de extensión durante 3 meses (escribiendo primero a partir de un formato y elaborando después un escrito para un contexto determinado, con todos los borradores y correcciones necesarios), que no que produzca en el mismo periodo de tiempo 5 redacciones de dos páginas cada una o (todavía más breves) 10 escritos de una página.

\section{FORMACIÓN EN ESTRATEGIAS DE COMPOSICIÓN}

Un aspecto importante en el tratamiento de la escritura en secundaria es la formación del aprendiz en las estrategias cognitivas de composición de textos. En otros textos (Cassany, Luna y Sanz, 1993) se desarrollan los contenidos lingüísticos de la escritura, en sus tres vertientes: procedimientos, conceptos y actitudes. En este apartado, me referiré a la formación del bloque de procedimientos, que afecta básicamente a las estrategias de composición.

La investigación sobre los procesos de composición de textos (Bereiter y Scardamalia, 1987; Cassany, 1987; Camps, 1994) ha demostrado la necesidad de formar los aprendices-autores en habilidades cognitivas específicas de escritura (buscar información, saber estructurarla, saber adaptarla al lector, etc.). La formación en estas habilidades puede realizarse a través de diferentes caminos. Por un lado, pueden enseñarse al aprendiz varias técnicas de escritura aplicables a los distintos procesos implicados en la creación de un texto. Por otro lado, pueden organizarse actividades de autoanálisis y reflexión para que el sujeto escritor incremente su control metacognitivo sobre el proceso de escritura, para que sea más consciente de su forma de escribir: de las estrategias que utiliza, de los bloqueos que experimenta, etc. Estas dos posibilidades metodológicas se complementan entre sí para desarrollar globalmente las habilidades del aprendiz.

La siguiente lista de técnicas aplicables a los diferentes procesos y subprocesos de composición es una selección personal hecha a partir de las propuestas de varios autores. Entre otros, Flower (1989) parece muy interesante:

1. Representarse la tarea de escritura: hacer un análisis del lector, hacerse una imagen del problema retórico, hacer un proyecto de texto, establecer un plan de trabajo, desarrollar un enunciado.

2. Generar ideas: torbellino de ideas, escritura automática, explorar un problema, desarrollar palabras clave, dialogar con el lector. 
3. Organizar ideas: mapa mental o conceptual, esquemas jerárquicos y decimales, clasificación de ideas en grupos.

4. Textualizar: concentrarse en aspectos parciales (ortografía, párrafos, léxico), marcar visualmente la prosa para el lector, usar marcadores discursivos, revelar el propósito y la estructura.

5. Evaluar y diagnosticar: oralizar el texto, simular la reacción del lector, comparar borrador con los planes iniciales y con el texto ideal (imaginado), aplicar guiones de autoevaluación de textos específicos (descripción, carta, informe), dialogar con un lector intermedio, colaborar con compañeros.

6. Operar cambios: reestructurar el texto, aplicar programas informáticos, pasar a limpio el texto, buscar economía y claridad en la prosa, adaptar la legibilidad al lector.

La aplicación en el aula de estas técnicas puede seguir la siguiente secuencia: el profesor explica con ejemplos y muestra en la práctica (experimentando él o ella el ejercicio ante la clase) cómo funciona cada técnica. A continuación, los aprendices aplican individualmente o en pequeños grupos el mismo procedimiento, según las características de cada técnica. La parte final de la secuencia puede destinarse a comentar las incidencias de esta y a intercambiar opiniones. Es pertinente recordar que no existen técnicas válidas o buenas en absoluto, sino que cada persona debe aplicarlas a su estilo personal de composición.

La segunda opción metodológica reseñada, que tiene el objetivo didáctico de incrementar el control metacognitivo, puede basarse en el uso de la oralidad (el diálogo entre aprendices, la verbalización individual, la entrevista entre aprendiz y profesor, etc.) para socializar y contrastar las habilidades mentales de cada uno. De acuerdo con los planteamientos vigotskyanos de desarrollo del lenguaje, la oralidad constituye un instrumento fundamental y poderoso para mejorar el control del lenguaje escrito.
Algunos de los ejercicios que pueden ser válidos para conseguir estos objetivos son: la redacción por parejas, que obliga a cada aprendiz a justificar ante el compañero sus opciones lingüísticas; los cuestionarios exploratorios, que indagan el comportamiento mental de cada autor y lo contrastan con el de sus compañeros, o el análisis de los borradores de un texto, así como de las correcciones y las modificaciones que realiza su autor durante todo el proceso de composición, los cuales contienen marcas y señales explícitos de los procesos internos que ha seguido el autor.

Vamos a terminar este apartado con un ejemplo concreto de formación de habilidades cognitivas: las destrezas de revisión de textos. La investigación sobre los procesos de composición (Bereiter y Scardamalia, 1987; Camps, 1994) ha identificado las operaciones que incluye la habilidad de revisar: evaluar o comparar el texto, que consiste en comparar el texto producido con los planes iniciales o con la imagen de texto ideal que tiene el autor; diagnosticar, que consiste en hallar los puntos susceptibles de modificación; y operar, que consiste en materializar los cambios y que consta de dos suboperaciones: elegir la táctica, esto es, decidir la forma idónea de llevar a cabo los cambios, y realizar el cambio.

Estas operaciones se refieren a las destrezas necesarias para que un aprendiz-autor pueda revisar sus propios textos. Pero la clase de lengua es una colectividad de aprendices, es -io debe ser!- un grupo de personas que colaboran en equipo para aprender y mejorar sus textos. Esta característica añade una dimensión nueva a la ya mencionada: el aprendiz debe tener la capacidad de poder revisar los textos de sus compañeros. Esto significa que, además de las habilidades individuales, el aprendiz debe desarrollar destrezas de interacción con sus compañeros para poder intercambiarse opiniones y para que cada uno pueda aprovecharlas en aras de mejorar sus textos. En la tabla 6 se presentan las destrezas específicas de revisión por parejas. 
Tabla 6. Destrezas de la revisión por parejas

\begin{tabular}{ll}
\hline \multicolumn{1}{c}{ Aprendiz-autor } & \multicolumn{1}{c}{ Aprendiz-lector } \\
\hline Saber escuchar al lector. & Saber comprender el escrito. \\
No interrumpirle. & Resumir, parafrasear, analizar, comentar. \\
Saber preguntar al lector. & Dar opiniones constructivas y útiles. \\
Aceptar las ideas del lector. & Atender las demandas del autor. \\
Ejercer la libertad de aceptar o no. & No imponer sus criterios. \\
\hline
\end{tabular}

Fuente: Elbow y Belanoff (1989).

Como se observa, estas destrezas se refieren a aspectos pragmáticos y lingüísticos de la interacción, como los turnos de habla (dejar hablar, no interrumpir), la modalidad de la intervención (hacer preguntas, atender las preguntas), el tono (opiniones constructivas) o los derechos de autor y lector (libertad de aceptar o no las críticas por parte del autor, deber de no imponer sus criterios por parte del lector).

Los ejercicios prácticos de revisión por parejas deben tener en cuenta estas destrezas. La tabla 7 ilustra un ejemplo que, además de intentar mejorar los textos de los aprendices, tiene el objetivo complementario de formar las habilidades mencionadas.

\section{EVALUACIÓN}

La medición de la capacidad de expresión escrita debe tener en cuenta tres grandes perspectivas: la calidad de los productos finales escritos del aprendiz; las estrategias cognitivas que este utiliza durante el proceso de composición, y las actitudes y los hábitos generales que tiene respecto a la práctica general de la escritura. Sea cual sea la finalidad de la evaluación (para diagnosticar el nivel previo, para medir el progreso alcanzado, o para determinar si el aprendiz ha conseguido un nivel predeterminado de competencia), esta debe tener en cuenta estos tres apartados, si pretende ser global.

Los instrumentos de evaluación que pueden utilizarse son muchos y variados, y dependen del apartado concreto que quiera evaluarse. Para la valoración del producto, es posible recurrir a baremos analíticos o sintéticos de análisis de escritos (Cassany, Luna y Sanz, 1994), fundamentados en los criterios pragmáticos, discursivos y lingüísticos más habituales (Cassany, 1993a): adecuación (selección del dialecto y del registro apropiados a la situación de escritura), coherencia (selección y estructuración de la información), cohesión (anáforas, progresión temática, puntuación, conectores, etc.), corrección (normativa) y variación o estilística.

En cambio, la evaluación del proceso y de las actitudes debe utilizar instrumentos más cualitativos, como las guías de observación, las entrevistas o el análisis de la carpeta, que pueden atender a aspectos más procedimentales y actitudinales, como las destrezas que usar el alumno o los valores y los hábitos que mantiene. En otras publicaciones se habla de las guías de observación (Cassany, 1993b) y de las entrevistas (Camps, 1994), de modo que aquí me centraré en la carpeta.

El análisis de la carpeta permite extraer mucha información sobre los procesos de composición y las actitudes del alumno. Los criterios básicos que guían el análisis son la cantidad de entradas recogidas, su cualidad, la progresión que demuestra el aprendiz-autor a lo largo del periodo de aprendizaje valorado y la diversidad de temas y textos que presenta. En la tabla 8 se detallan estos criterios.

En resumen, las carpetas pueden proporcionar mucha información sobre el proceso de composición y las actitudes que tiene el aprendiz. Se trata de un instrumento fundamental de evaluación formativa en este nuevo planteamiento de la enseñanza de la escritura en secundaria. 
Tabla 7. Equipo de revisión

Equipo de revisión es un ejercicio de trabajo en grupo en el que los aprendices se ayudan entre sí para mejorar sus escritos. Forma un equipo con 2 o 3 compañeros y sigue las siguientes instrucciones:

\begin{tabular}{|c|c|}
\hline Aprendiz-autor & Aprendices-lectores \\
\hline $\begin{array}{l}\text { Lee en voz alta tu texto. Hazlo despacio, sin prisas. Léelo } \\
\text { dos veces, si es necesario. Deja tiempo para que tus compa- } \\
\text { ñeros lo entiendan. }\end{array}$ & $\begin{array}{l}\text { Lee y escucha activamente el texto de tu compañero. Léelo dos } \\
\text { o más veces, si hace falta. Anota todo lo que te llame la atención } \\
\text { (palabras, frases, ideas, etc.). ¿Qué te parece el texto?, ¿lo has } \\
\text { entendido todo?, ¿qué cambiarías? }\end{array}$ \\
\hline Escucha los comentarios. Pregunta lo que no comprendas. & Explica al autor tus impresiones. \\
\hline Da tu opinión. ¿Estás de acuerdo? & Escucha la opinión del autor. Pregunta lo que no entiendas. \\
\hline $\begin{array}{l}\text { Relee lentamente el texto con los compañeros. Párate en los } \\
\text { puntos mejorables y haz los cambios necesarios. }\end{array}$ & $\begin{array}{l}\text { Relee poco a poco el texto con el autor. Párate en los puntos } \\
\text { mejorables y sugiere los cambios necesarios. }\end{array}$ \\
\hline $\begin{array}{l}\text { Consejos } \\
\text { - No interrumpas a los compañeros. Escucha sus opiniones } \\
\text { e intenta comprenderlas. } \\
\text { - Debes ser positivo y respetuoso cuando hables de tu texto } \\
\text { y de los comentarios de tus compañeros. } \\
\text { - Asume todo lo que pueda mejorar el texto. } \\
\text { - No estás obligado a aceptar lo que digan los compañeros. } \\
\text { Tú decides qué aceptas y qué no. }\end{array}$ & $\begin{array}{l}\text { Consejos } \\
\text { • Debes ser constructivo y respetuoso. Señala los puntos fuertes } \\
\text { y los débiles. } \\
\text { • Haz comentarios específicos, que ayuden a mejorar el escrito. } \\
\text { Evita expresiones generales como: "Está bien!", "iMe gusta!”, } \\
\text { que ayudan poco. Comenta punto por punto. } \\
\text { - No impongas tu opinión a los demás. El autor tiene libertad } \\
\text { para hacer lo que crea conveniente. }\end{array}$ \\
\hline
\end{tabular}

Fuente: elaboración propia.

Tabla 8. Criterios para la evaluación de la carpeta

A. Cantidad de entradas. Se puede verificar:

- Si el aprendiz ha producido todos los textos que el profesor había establecido.

- Si el aprendiz ha tomado la iniciativa y ha escrito espontáneamente, sin que haya habido instrucción del profesor.

- Si el aprendiz elabora muchas, pocas o ninguna producción intermedia y qué tipo de producciones son (borradores, esquemas, correcciones, etc.).

- Si las fechas de las entradas se reparten durante todo el periodo evaluado o, al contrario, se concentran en un pequeño lapso de tiempo, lo cual indicaría si ha habido un trabajo continuado de escritura o no.

B. Cualidad de las entradas. Se puede considerar:

- Si el aprendiz utiliza espontáneamente técnicas concretas de composición, estudiadas en clase o no, como las mencionadas en el apartado anterior.

- Si el aprendiz realiza modificaciones en las diversas producciones intermedias. Se puede verificar hasta qué punto el aprendiz usa las estrategias de composición para mejorar el producto escrito final.

- Si el aprendiz atiende a las sugerencias de mejora que le realiza el profesor en las entrevistas o a las que pueden plantear los compañeros en las actividades de revisión por parejas o pequeños grupos.

- $\quad$ Si el aprendiz consulta manuales de referencia (gramáticas, diccionarios) para mejorar sus producciones.

- Si el aprendiz usa tecnología informática para producir textos y que tipo de software (procesadores de texto, verificadores ortográficos, diccionarios digitales, verificadores de estilo, etc.).

- Si el aprendiz colabora con sus compañeros en actividades de escritura (planificación, textualización, revisión) y si saca provecho de esta colaboración.

C. Diversidad de temas, textos, tonos, etc.

- $\quad$ Si el aprendiz escribe sobre temas variados (académicos, personales, sociales, etc.), si usa varios tipos de texto, si se arriesga a usar registros, estilos y recursos lingüísticos diversos.

- Si el aprendiz usa estrategias de composición diferentes según las características de la situación de comunicación (tema, tipo de texto, destinatario, función, etc.).

D. Progresión

- Si el aprendiz incrementa la cantidad de entradas a lo largo del periodo evaluado, y si mejora la calidad lingüística de los escritos.

- $\quad$ Si el aprendiz incrementa el número de producciones intermedias para generar un solo texto.

- Si el aprendiz diversifica temas, tipos de texto y estilos.

Fuente: elaboración propia. 


\section{REFERENCIAS BIBLIOGRÁFICAS}

Bereiter, C. y Scardamalia, M. (1987). The Psychology of Written Composition. Hillsdale, N.J.: Erlbaum.

Bosch, M. (1996). Autonomia $i$ autoaprenentatge de Ilengües. Barcelona: Graó.

Calfee, R.C. y Perfumo, P. (1993). Carpetas de estudiante: oportunidades para una revolución en la evaluación. CLE, 19-20, 87-96.

CAMPs, A. (1994). L'ensenyament de la composició escrita. Barcelona: Barcanova.

Cassany, D. (1987). Descriure escriure. Com s'aprèn a escriure. Barcelona: Empúries. Versión castellana: Describir el escribir. Cómo se aprende a escribir. Barcelona: Paidós. 1989.

Cassany, D. (1993a). Reparar l'escriptura. Barcelona: ICE UB/Graó. Versión castellana: Reparar la escritura. Barcelona: Graó. 1993.

CASSANy, D. (1993b). La cuina de l'escriptura. Barcelona: Empúries. Versión castellana: La cocina de la escritura. Barcelona: Anagrama. 1995.

Cassany, D.; Luna, M. y Sanz, G. (1993). Ensenyar llengua. Barcelona: Graó. Versión castellana: Enseñar lengua. Barcelona: Graó. 1994.

Elbow, P. y BelanofF, P. (1989). Sharing and Responding. Nueva York: Random House.

Flower, L. (1989). Problem Solving Strategies for Writing. Orlando: Harcourt Brace Jovanovich. $3^{\text {a }}$ ed.

FREINET, C. (1968). La méthode naturelle. I. L'apprentissage de la langue. Neuchâtel: Delachaux et Niestlé. Versión castellana: Los métodos naturales. I. El aprendizaje de la lengua. Barcelona: Fontanella, 1970.

Freinet, C. (1969). Conseils aux jeunes. Cannes: Coopérative de I'Enseignement Laïc.

Fulwiler, T. (1987) Teaching With Writing. Portsmouth: Heinemann.
Fulwiler, T. y Young, A. (1990) Programs That Work. Models and Methods for Writing Across the Curriculum. Portsmouth: Heinemann.

GILL, K. (ed.) (1993). Process and Portfolios in Writing Instruction. Classroom Practices in Teaching English, 26. NCTE Committee on Classroom Practices.

Holec, H. (ed.) (1988). Autonomy and Self-Directed Learning. Present Fields of Application. Proyecto No. 2. Estrasburgo: Consejo de Europa.

Jakobson, R. (1963). Essais de Lingüistique Générale. París: Éditions du Minuit.

MunBY, J. (1968). Read and Think: Training in Intensive Reading Skills. Londres: Longman.

Murphy, S. y SMith, M.A. (1992). Writing Portfolios. A Bridge from Teaching to Assessment. Markham (Ontario): Pippin Publishing Lmd.

REID, J. (1994). Responding to ESL Students' Texts: The Myths of Appropriation. TESOL Quarterly, 28(2), 273-292.

Ribé, R. y Dejuan, E. (1984) 'Extensive reading activities' a BUP i COU. Una experiència de planificació i sistematització. Papers de Batxillerat, 5, 81-87.

SÁnCHEZ, E., J. y RINCÓN, F. (1985) Los talleres literarios. Una alternativa didáctica al historicismo. Barcelona: Montesinos.

VALENCIA, Sh.W. (1993). Método de carpeta para la evaluación de la lectura en clase: los porqué, los qué y los cómo. CLE, 19-20, 69-75.

WeLLS, G. (1987). Aprendices en el dominio de la lengua escrita. En: Psicología y Educación. Realizaciones y tendencias en la investigación y en la práctica (pp. 5772). Actas de las II Jornadas Internacionales de Psicología y Educación. Madrid: Visor-Aprendizaje/MEC.

WHITE, R.V. (1983). Approaches to the teaching of reading. Teaching Language as Communication (pp. 42-53). Primeres Jornades Pedagògiques d'Anglès. Barcelona: ICE-UAB. 